\title{
CARACTERÍSTICAS HISTOLÓGICAS Y ENDOSCÓPICAS DEL CÁNCER GÁSTRICO DIAGNOSTICADO EN UN HOSPITAL NACIONAL DEL CALLAO, PERÚ
}

\author{
Briny Rodríguez-Vargas ${ }^{1,2, a}$, Fernando Arévalo-Suarez ${ }^{1,3, b}$, Eduardo Monge-Salgado ${ }^{1,2, c}$, \\ Pedro Montes-Teves ${ }^{1,3, c}$
}

RESUMEN

Objetivos. Describir las características histológicas y endoscópicas que se presentan en pacientes diagnosticados con cáncer gástrico en el Hospital Nacional Daniel Alcides Carrión del Callao. Materiales y métodos. Se realizó un estudio tipo serie de casos que incluyó a todos los pacientes con diagnóstico histológico de cáncer gástrico durante el periodo de enero de 2009 a diciembre de 2011. La información se obtuvo de los registros del servicio de anatomía patológica del Hospital Nacional Daniel Alcides Carrión. Se consignó la edad y sexo de los pacientes, el tipo histológico, la localización endoscópica, así como la presencia de metaplasia intestinal, el grado histológico y la morfología del cáncer. Resultados. Se incluyeron 120 pacientes. La edad promedio fue de 65,4 \pm 13,6 años; 59 (49\%) fueron de sexo masculino. Según el tipo histológico se encontró el tipo intestinal en 68 (56\%); difuso en $45(38 \%)$, y mixto en 7 (6\%). Según su localización, 23 (19\%) se localizaron en fondo; 52 (43\%) en el cuerpo; 39 (33\%) en el antro, y 6 (5\%) en el píloro. Los pacientes con cáncer gástrico de tipo intestinal presentaron una edad promedio mayor que los que tuvieron el tipo difuso $(69,1 \pm 10,3$ versus $59,3 \pm 15,3)$. De los pacientes con cáncer gástrico tipo intestinal, $60,3 \%$ tuvo localización proximal, en comparación a $66,6 \%$ de los pacientes con tipo difuso. Conclusión. En la población en estudio, el cáncer gástrico de tipo difuso se presenta a una edad más temprana que el de tipo intestinal, además de estar más frecuentemente localizados a nivel proximal.

Palabras clave: Cáncer gástrico; Endoscopía; Histología; Patología; Metaplasia (fuente: DeCS BIREME).

\section{HISTOLOGIC AND ENDOSCOPIC CHARACTERISTICS OF GASTRIC CANCER DIAGNOSED IN A NATIONAL HOSPITAL OF CALLAO, PERU}

\begin{abstract}
Objetivos. To describe the histologic and endoscopic characteristics reported among patients diagnosed with gastric cancer in "Daniel Alcides Carrion" National Hospital in Callao. Materials and methods. We performed a case series including all patients with histological diagnosis of gastric cancer from January 2009 to December 2011. Data were obtained from the registers of the pathology service of Daniel Alcides Carrion National Hospital. Factors such as age and gender of patients, histologic type, endoscopic location, presence of intestinal metaplasia, histologic degree, and cancer morphology were evaluated. Results. 120 patients were included. Mean age was $65.4 \pm 13.6$ years; $59(49 \%)$ were male. Based on the histologic type, intestinal type was found among 68 (56\%); diffuse type among $45(38 \%)$, and a mixed type in $7(6 \%)$. Regarding the site, $23(19 \%)$ of gastric cancers were located in the fundus; $52(43 \%)$ in the body; $39(33 \%)$ in the antrum, and $6(5 \%)$ in the pylorus. Patients with gastric cancer of the intestinal type were in average older than those with a diffuse type $(69.1 \pm 10.3$ versus $59.3 \pm 15.3) .60 .3 \%$ of intestinal-type gastric cancers were located proximally, versus $66.6 \%$ of diffuse-type cancers. Conclusion. Among the studied population, diffuse-type gastric cancer appears at an earlier age than the intestinal type, and its most common location is proximal.
\end{abstract}

Key words: Stomach neoplasms; Endoscopy; Histology; Pathology; Metaplasia (source: MeSH NLM).

\footnotetext{
Hospital Nacional Daniel Alcides Carrión. Callao, Perú.

Facultad de Medicina Humana "San Fernando", Universidad Nacional Mayor de San Marcos. Lima, Perú.

Facultad de Medicina, Universidad Peruana de Ciencias Aplicadas. Lima, Perú.

Médico cirujano; ${ }^{\mathrm{b}}$ médico anatomopatólogo; ${ }^{\mathrm{c}}$ médico gastroenterólogo

Recibido: 18-02-13 Aprobado: 06-03-13
}

Citar como: Rodríguez-Vargas B, Arévalo-Suarez F, Monge-Salgado E, Montes-Teves P. Características histológicas y endoscópicas del cáncer gástrico diagnosticado en un hospital nacional del Callao, Perú. Rev Peru Med Exp Salud Publica. 2013;30(1):12-7. 


\section{INTRODUCCIÓN}

El cáncer gástrico es el cuarto cáncer más frecuente a nivel mundial, detrás del cáncer de pulmón, mama y colorrectal, según estimaciones, en el año 2008 se registraron 988000 casos, lo cual representa un $7,8 \%$ del total ${ }^{(1)}$. De estos, más del $70 \%$ ocurren en países en desarrollo. En el Perú, el cáncer gástrico representa la mayor causa de morbilidad y mortalidad por cáncer en ambos sexos teniendo entre 2002-2007, 5215 casos, que representa el $13,3 \%$ del total de cánceres ${ }^{(2)}$.

La clasificación histológica más extendida es la propuesta por Lauren en $1965^{(3)}$, que distingue: a) El adenocarcinoma gástrico de tipo intestinal, caracterizado por la presencia de estructuras glandulares con distintos grados de diferenciación y que correspondería a los tipos tubular y papilar según la OMS, además a este tipo de cáncer gástrico se lo asocia con la infección de Helicobacter pylori; y b) Carcinoma gástrico difuso, caracterizado por la presencia de células que infiltran la pared gástrica sin formación glandular, con pobre grado de diferenciación, y mostrando en ocasiones la presencia de células "en anillo de sello", lo cual correspondería a los tipos anillo de sello y mucinoso según la OMS ${ }^{(3,4)}$.

Tradicionalmente, la localización más frecuente del cáncer gástrico es la región antropilórica (cáncer gástrico distal) ${ }^{(5,6)}$, el cual se relaciona con el tipo histológico intestinal. Sin embargo, en las últimas décadas se ha observado un incremento de la frecuencia de los tumores de cuerpo y fondo (cáncer gástrico proximal) y de la unión esófago-gástrica (7-9), lo cual se sospecha está asociado al cambio en la prevalencia también del tipo histológico de cáncer gástrico hacia el tipo difuso ${ }^{(10,11)}$.

En nuestro país un estudio mostró que el tipo difuso era el más frecuente $(51,5 \%)$, seguido del intestinal $(33,9 \%)^{(12)}$. Esto, sospechado en base a la disminución de la incidencia de cánceres de tipo intestinal. Además, se encontró asociación entre el tipo intestinal con la localización distal $(58,3 \%$ frente a $44,1 \%)$, y el tipo difuso con la localización proximal (19,3\% frente a $12,5 \%)$. Este estudio no es el único que ha mostrado que el tipo histológico tendría relación con la localización topográfica del cáncer gástrico, además de describir un aumento en la frecuencia del cáncer gástrico de tipo difuso. Sin embargo, se requieren más estudios locales para comprobar si esta variación de frecuencias es sostenida. Por todo ello, nuestro objetivo es describir las características histopatológicas y endoscópicas de los pacientes diagnosticados con cáncer gástrico en el Hospital Nacional Daniel Alcides Carrión.

\section{MATERIALES Y MÉTODOS}

Se realizó un estudio descriptivo tipo serie de casos en el que se incluyeron a todos los pacientes con diagnóstico histológico de carcinoma gástrico durante el periodo de enero de 2009 y diciembre de 2011 en el Hospital Nacional Daniel Alcides Carrión de la Provincia Constitucional del Callao en Perú, hospital de referencia del Ministerio de Salud para dicha región.

Se realizó una búsqueda en los archivos del Servicio de Anatomía Patológica, así como una nueva lectura y revisión de todas las láminas para confirmar el diagnóstico y tipo histológico. Los datos de las características endoscópicas se obtuvieron mediante la búsqueda y revisión de los informes endoscópicos en el archivo del Servicio de Gastroenterología.

Se excluyó a los pacientes cuyos archivos endoscópicos hayan estado incompletos o extraviados, que el diagnóstico haya estado errado con la relectura de las láminas, o la lámina haya sido extraviada. Así también, se excluyó a los pacientes que hayan tenido cáncer de localización en la unión gastroesofágica y los que tenían un informe histopatológico de neoplasia indiferenciada.

Dentro de las variables incluidas en el estudio se tomó en cuenta la edad y el sexo de los pacientes; el tipo histológico, según la clasificación de Lauren; además, se determinó la presencia de metaplasia intestinal (ausente, completa, incompleta, mixta o no evaluable), y el grado histológico (bien, mediana o pobremente diferenciado). En lo referente a las características endoscópicas, se determinó la localización en base al punto de extensión más alto (fondo, cuerpo, antro y píloro), y la morfología en base a la clasificación de Bormann (I, II, III, IV, V) y precoz.

La recolección de datos se realizó mediante el instrumento elaborado con base en las variables del estudio. La digitalización de los datos se realizó en el programa Excel 8.0 y el análisis con SPSS 13.0. Se analizó la distribución de la frecuencia de cáncer gástrico de acuerdo a la edad, con un intervalo de cada 10 años, y el tipo histológico. 


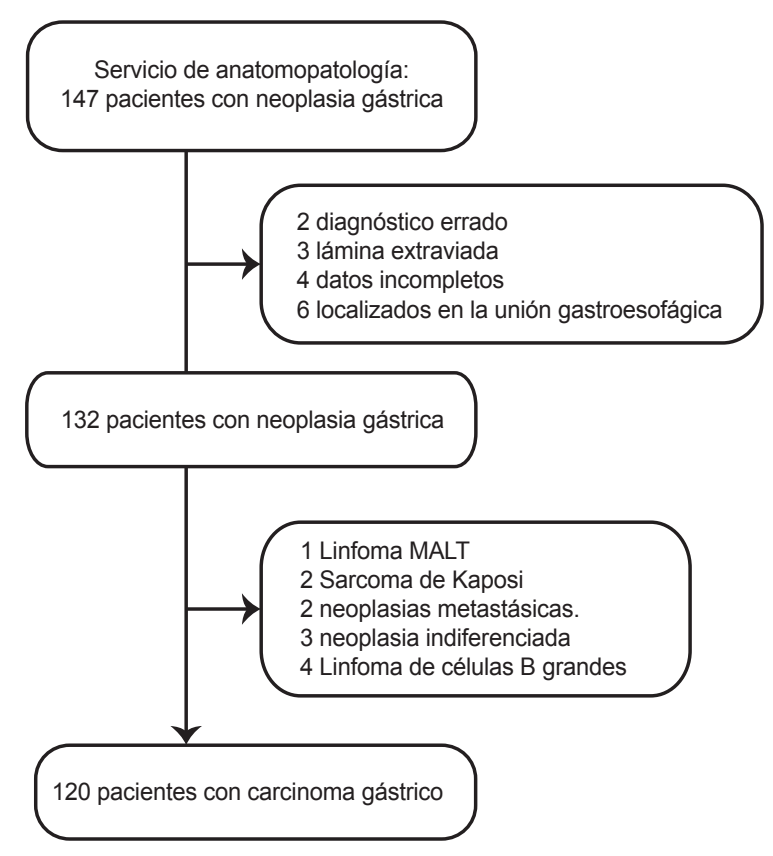

Figura 1. Flujograma de selección de pacientes.

El estudio no involucró ningún riesgo para los pacientes y se hizo empleando información rutinaria del Servicio de Gastroenterología, manteniendo los datos de forma confidencial.

\section{RESULTADOS}

Durante el período enero de 2009 a diciembre de 2011, se incluyeron inicialmente a 147 pacientes, informados como neoplasias gástricas por el Servicio de Anatomopatología; de ellos se excluyó a 27, como se observa en la Figura 1.

Finalmente, el análisis se realizó con 120 (91,8\%) pacientes con diagnóstico de carcinoma gástrico. El promedio de edad fue de $65,4 \pm 13,5$ años. Los pacientes de sexo masculino fueron 59 (49\%).

En cuanto a la localización del cáncer, 23 (19\%) se localizaron en fondo; 52 (43\%) en cuerpo; 39 (33\%) en el antro, y $6(5 \%)$ en el píloro. De acuerdo a la morfología se encontró $11(9 \%)$ pacientes con cáncer gástrico precoz; $9(8 \%)$ tipo Bormann I; $8(7 \%)$ tipo Bormmann II; 40 (33\%) tipo Bormann III; 10 (8\%) tipo Bormann IV, y 42 (35\%) tipo Bormann V.
Tabla 1. Características histológicas y endoscópicas de acuerdo a los tipos histológicos de cáncer gástrico. Hospital Nacional Daniel Alcides Carrión, Perú.

\begin{tabular}{lccc}
\hline & $\begin{array}{c}\text { Intestinal/tubular } \\
\mathbf{n}(\%)\end{array}$ & $\begin{array}{c}\text { Difuso/anillo } \\
\text { de sello n(\%) }\end{array}$ & $\begin{array}{c}\text { Mixto } \\
\mathbf{n}(\%)\end{array}$ \\
\hline Metaplasia intestinal & & & \\
\hline Ausente & $37(55)$ & $36(80)$ & $3(43)$ \\
\hline Incompleta & $3(4)$ & $1(2)$ & $0(0)$ \\
\hline Completa & $17(25)$ & $6(14)$ & $4(57)$ \\
\hline Mixta & $5(7)$ & $1(2)$ & $0(0)$ \\
\hline No evaluable & $6(9)$ & $1(2)$ & $0(0)$ \\
Grado histológico & & & \\
\hline Bien diferenciado & $12(18)$ & $0(0)$ & $0(0)$ \\
\hline Pobre diferenciado & $18(26)$ & $42(93)$ & $6(85)$ \\
\hline Medianamente & $38(56)$ & $3(7)$ & $1(16)$ \\
diferenciado & & & \\
Localización & $9(13)$ & $12(27)$ & $2(29)$ \\
\hline Fondo & $32(47)$ & $18(40)$ & $2(29)$ \\
\hline Cuerpo & $22(32)$ & $15(33)$ & $2(29)$ \\
\hline Antro & $5(8)$ & $0(0)$ & $1(14)$ \\
\hline Píloro & & & \\
\hline Morfología & $3(5)$ & $7(15)$ & $0(0)$ \\
\hline Precoz & $25(37)$ & $16(36)$ & $1(14)$ \\
\hline Bormann I & $8(12)$ & $1(2)$ & $0(0)$ \\
\hline Bormann II & $5(7)$ & $3(7)$ & $0(0)$ \\
\hline Bormann III & $20(29)$ & $15(33)$ & $5(72)$ \\
\hline Bormann IV & & & \\
\hline Bormann V & & & \\
\hline & & $14)$ \\
\hline
\end{tabular}

De acuerdo al tipo histológico se halló pacientes con cáncer gástrico tipo intestinal: 68 (56\%); tipo difuso: 45 (38\%), y tipo mixto: 7 (6\%). En lo referente al grado de diferenciación se encontró lo siguiente: bien diferenciado: 13 (10,8\%); medianamente diferenciado: $41(34,2 \%)$, y pobremente diferenciado: 66 (55\%). La metaplasia intestinal estuvo ausente en el $63 \%$ de los casos, incompleta en $3 \%$, completa en $23 \%$, mixta en $5 \%$, y no evaluable en $6 \%$.

La edad promedio de los pacientes con cáncer gástrico tipo intestinal fue de 69,1 \pm 10,3 años; de los de tipo difuso fue $59,3 \pm 15,3$ años y de los de tipo mixto $69,1 \pm 17,1$ años.

Respecto al grado de diferenciación histológica, se encontró un $93 \%$ pobremente diferenciados en los pacientes con cáncer gástrico tipo difuso, comparado con un $26 \%$ en el tipo intestinal, así como $18 \%$ de bien diferenciados en el intestinal contra $0 \%$ en el difuso. Las características histológicas se resumen en la Tabla 1. 
En lo referente a la característica endoscópica del Bormann, los más hallados fueron el Bormann III y $V$ siendo los resultados similares entre ambos tipos de cáncer gástrico (Tabla 1).

En total $14 \%$ (17) de los casos ocurrieron en pacientes menores de 50 años, de estos, 14 $(82 \%)$ fueron pacientes con carcinoma gástrico tipo difuso.

En los pacientes con cáncer gástrico tipo intestinal, la localización más freceunte fue proximal con $41(60,3 \%)$ pacientes; mientras que $30(66,6 \%)$ de los pacientes de tipo difuso tuvieron dicha localización.

\section{DISCUSIÓN}

De todas las neoplasias gástricas, aproximadamente el $95 \%$ constituyen carcinomas ${ }^{(13,14)}$ en nuestro estudio se encontró $91,8 \%$ de carcinomas gástricos lo cual va de acuerdo a lo tradicionalmente encontrado. De los 120 casos encontrados en los tres años analizados el promedio de edad fue de 65,4 años lo cual está de acuerdo con lo descrito por otras serias peruanas como la de Quiñones J. et al. (12), en la cual el promedio de edad fue el mismo; sin embargo, se evidencia diferencia en la distribución por sexo, pues reportan $62,4 \%$ en sexo masculino, mientras que en este estudio fue de $49,1 \%$.

Además, se encontraron diferencias de edad entre los pacientes con cáncer de tipo intestinal y difuso, lo cual sugiere la presentación en edades más tempranas del cáncer gástrico tipo difuso, particularmente en la población menor de 50 años, en la cual se encontró $17(14 \%)$ casos de cáncer gástrico, de los cuales 14 (83\%) fueron tipo difuso. Esto estaría en relación a los diferentes mecanismos de carcinogénesis propuestos, ya que el cáncer gástrico de tipo difuso no estaría en relación con los cambios producidos por la infección por Helicobacter pylori sino por factores genéticos (mutaciones gen de E-cadherina) (15-19) y, por ende, el tiempo de progresión a cáncer gástrico seria más corto.

En la mayoría de las series descritas, el tipo intestinal es el predominante, por ejemplo, en el estudio de Borch (16), el tipo intestinal representó el 52,7\%, seguido del difuso con un $33,4 \% \mathrm{y}$, por último, el mixto con un $13,9 \%$. En el Perú, Cienedes et al. ${ }^{(17)}$ en un estudio realizado en un hospital nacional de
Lima, encontraron un $66,9 \%$ para el tipo intestinal, un $27 \%$ para el tipo difuso y un $6,1 \%$ para el mixto. En el estudio reciente de Quiñones J. et al. (12), el tipo difuso fue más frecuente $(51,5 \%)$, seguido del intestinal $(33,9 \%)$ y, por último, el mixto con un $14,6 \%$. Esto sospechado en base a la disminución de la incidencia de cánceres de tipo intestinal. Sin embargo, en este estudio se muestra lo publicado en la mayoría de las series con cánceres gástricos tipo intestinal 68 (56,7\%); difuso $45(37,5 \%)$, y Mixto $7(5,8 \%)$ y también se asemeja a un estudio colombiano publicado recientemente por Martínez J. et al. ${ }^{(18)}$ en donde se encontró $53 \%$ de intestinal y $47 \%$ de difuso. Lo cual indica todavía una mayor proporción de cáncer gástrico del tipo intestinal en nuestro hospital, aunque se observa un mayor número de cánceres gástricos de tipo difuso.

Tradicionalmente es conocida la distribución topográfica del cáncer gástrico teniendo entre un $40-60 \%$ de los casos localizados en antro-píloro, un $20-25 \%$ en cuerpo y un $15-20 \%$ en fondo $y$ cardias (14); como en el estudio de Pilco et al. (19), donde encontraron $68 \%$ de cánceres gástricos de localización distal y $32 \%$ de proximales. Recientemente ha habido algunas publicaciones que han mostrado un cambio en esta localización como la de Quiñones J. et al. (12), en donde se encontró asociación entre el tipo intestinal con la localización distal $(58,3 \%$ frente a $44,1 \%$; $p=0,004)$, y el tipo difuso con la localización proximal (19,3\% frente a $12,5 \%$; $p=0,049$ ); o en el estudio de Martínez J. et al. ${ }^{(18)}$ donde el $56,4 \%$ de los cánceres fueron proximales y $43,6 \%$ distales.

En el presente estudio se confirma el cambio en la localización del cáncer gástrico pues se encuentra que el $62 \%$ fueron proximales $y$ el $38 \%$ fueron distales; sin embargo, en la distribución entre intestinal y difuso no hay diferencia, encontrándose que el $60 \%$ de los intestinales y el $66 \%$ de los difusos son proximales. No obstante, deberían de realizarse estudios prospectivos, de mayor duración, con mayor tamaño muestral y multicéntrico a fin de poder generalizar esta aseveración. Además, al no ser este hospital un centro de referencia de nivel nacional, lo presentes hallazgos no podrían ser generalizados a toda la población peruana, pero sí podrían ser tomados en cuanta para dar un acercamiento de las características de los pacientes con cáncer gástrico en la provincia del 
Callao al ser este hospital el centro de referencia del Ministerio de Salud en dicha región.

En conclusión, se encontró que el cáncer gástrico de tipo difuso se presenta a una edad más temprana que el tipo intestinal. El tipo más frecuente de cáncer gástrico es aún el intestinal; sin embargo, se observó un aumento de los casos de cáncer gástrico de tipo difuso. Se puede afirmar que en nuestro hospital la localización del cáncer gástrico tanto de tipo intestinal como difuso es más frecuente a nivel proximal, lo cual podría tener influencia en el tratamiento y pronóstico. Además, no se encontró relación entre el tipo histológico y la localización del cáncer gástrico.

Contribuciones de autoría: todos los autores han participado en la concepción del artículo, su redacción y aprobación de la versión final. Además, BRV realizó la recolección de datos, el análisis de datos, diseño del artículo, obtención de los resultados. FAS contribuyó con la selección de pacientes y con la re-lectura de las láminas de anatomopatologia; y PMT participó en el análisis e interpretación de datos.

Fuentes de financiamiento: autofinanciado.

Conflictos de interés: los autores declaran no tener conflictos de interés.

\section{REFERENCIAS BIBLIOGRÁFICAS}

1. Ferlay J, Shin HR, Bray F, Forman D, Mathers C, Parkin DM. Globocan 2008. V2.0, Cancer incidence and Mortality Worldwide: IARC Cancer Base No 10 [Internet]. Lyon: International Agency for Research on Cancer; 2010 [citado el 15 de agosto del 2012]. Disponible en: http:// globocan.iarc.fr/

2. Perú, Ministerio de Salud, Dirección General de Epidemiología. Análisis Situacional de Salud del Perú 2010. Lima: MINSA; 2010.

3. Lauren P. The two histological main types of gastric carcinoma: diffuse and so-called intestinaltype carcinoma. An attempt at a histo-clinical classification. Acta Pathol Microbiol Scand 1965;64:31-49.

4. Fenoglio-Preiser C, Muñoz N, Carneiro F, Powell SM, Correa P, Rugge $\mathrm{M}$ et al. Gastric carcinoma. En: Hamilton SR, Aaltonen LA, editors. World Health Organization Classification of Tumours. Pathology \& Genetics. Tumours of the digestive system. Lyon: IARC Press; 2000. p. 37-68.

5. Buruk F, Berberoglu U, Pak I, Aksaz E, Celen O. Gastric Cancer and Helicobacter pylori infection.
Br J Surg. 1993;80(3):378-9.

6. Martín-de-Argila C, Boixeda D, Redondo C, Alvarez I, Gisbert JP, García Plaza A, et al. Relation between histologic subtypes and location of gastric cancer and Helicobacter pylori. Scand J Gastroenterol. 1997;32(4):303-7.

7. Calli Demirkan N, Tunçyürek M, Ugur Ertan E, Bülent Alkanat M, Içöz G. Comparaison histopronostique

des classifications des carcinomes gastriques en fonction de leur localisation. Gastroenterol Clin Biol 2002;26(6-7):610-5.

8. Yu J, Zhao Q. The demographic characteristics of histological types of gastric cancer with gender, age, and tumor location. J Gastrointest Canc. 2009;40(3-4):98-100.

9. Blot WJ, Devesa SS, Kneller RW, Fraumeni JF Jr. Rising incidence of adenocarcinoma of the esophagus and gastric cardia. JAMA 1991;265(10):1287-9.

10. Antonioli DA, Goldman H. Changes in the location and type of gastric adenocarcinoma. Cáncer 1982;50(4):775-81.

11. Kampschöer GH, Nakajima T, van de Velde CJ. Changing patterns in gastric adenocarcinoma. Br J Surg. 1989;76:914-6.

12. Quiñones J, Portanova M, Yabar A. Relación entre Tipo Histológico y la Localización del Adenocarcinoma Gástrico en el Hospital Rebagliati. Rev Gastroenterol Peru. 2011;31(2):139-45

13. Layke JC, Lopez PP. Gastric cancer: diagnosis and treatment options. Am Fam Physician. 2004;69(5):1133-40.

14. Quintero Carrión E, Nicolás Pérez D. Adenocarcinoma gástrico y de la unión esófago-gástrica. Medicine 2004;9(2):100-11.

15. Chen HC, Chu RY, Hsu PN, Hsu PI, Lu JY, Lai KH, et al. Loss of E-cadherin expression correlates with poor differentiation and invasion into adjacent organs in gastric adenocarcinomas. Cancer Letters. 2003;201(1):97-106.

16. Borch K, Jönsson B, Tarpila E, Franzén T, Berglund J, Kullman $\mathrm{E}$, et al. Changing pattern of histological type, location, stage and outcome of surgical treatment of gastric carcinoma. British Journal of Surgery. 2000;87(5):618-26. 
17. Cienedes D, Cok J, Bussalleu A. Tipos histológicos de Cáncer Gástrico de acuerdo a la clasificación de Lauren en el Hospital Nacional Cayetano Heredia. Rev Gastroenterol Peru. 1995; 15(3):239-46.

18. Martinez J, Garzon M. Características de los pacientes con cáncer gástrico del departamento de Cundinamarca, remitidos al Hospital Universitario de la Samaritana entre los años 2004 y 2009. Rev Col Gastroenterol. 2010;25(4):344-8.

19. Pilco P, Viale S, Ortiz N. Cáncer gástrico en un hospital general:
Hospital Santa Rosa. Rev Gastroenterol Peru. 2009;29(1):66-74.

Correspondencia: Briny Rodríguez Vargas Dirección: Jr. Inca Yupanqui 474-Urbanizacion Tahuantinsuyo. Lima 28, Perú. Teléfono: (511) 978490239

Correo electrónico:borv1905@gmail.com

Visite nuestra página en Facebook, www.facebook.com/rpmesp Infórmese sobre los eventos y los nuevos contenidos de la Revista Peruana de Medicina Experimental y Salud Pública

\section{REVISTA PERUANA DE MEDICINA EXPERIMENTAL Y SALUD PÚBLICA}

Difundiendo investigación en salud $1942-2012$
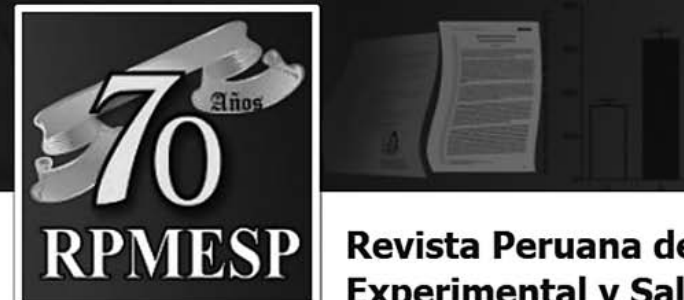

Revista Peruana de Medicina Experimental y Salud Pública (OFICIAL)

A 578 personas les gusta esta página $\cdot 13$ personas están hablando sobre esto

Comunidad

La Revista Peruana de Medicina Experimental y Salud Publica es el órgano oficial de difusión científica del Instituto Nacional de Salud

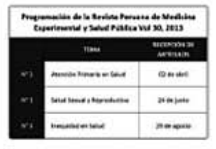

Fotos

\section{8}

Me gusta
COMENTARIOS DF SIMPOSIO SALUD El simposio de verda estuvo muy bueno. $\mathrm{N}$

Notas 5 